\title{
Study the Relation Between Acetylcholinesterase and Obesity in University Students
}

\author{
Asmaa Fathi Hamouda 1, 2, 3, *, Ibrahim Abdu Khardali ${ }^{3}$, Ibraheem Mohammed Attafi ${ }^{3}$, \\ Magbool Essa Oraiby ${ }^{3}$, Mohammad Ahmad Attafi ${ }^{3}$, Ali Mousa Sulaiman Muyidi ${ }^{3}$, \\ Hassan Abdu Ahmed Dohali ${ }^{4}$ \\ ${ }^{1}$ Department of Biochemistry, Faculty of Science, University of Alexandria, Alexandria, Egypt \\ ${ }^{2}$ Medical Laboratory Technology Department, Faculty of Applied Health Sciences, Jazan University, Jazan, Kingdom of Saudi Arabi \\ ${ }^{3}$ Poison Control and Medical Forensic Chemistry Center, Ministry of Health, Jazan, Kingdom of Saudi Arabia \\ ${ }^{4}$ Ministry of Health, Abu-Arish General Hospital Jazan, Jazan, Kingdom of Saudi Arabia
}

Email address:

asmaakingdom1@yahoo.com (A. F. Hamouda)

${ }^{*}$ Corresponding author

\section{To cite this article:}

Asmaa Fathi Hamouda, Ibrahim Abdu Khardali, Ibraheem Mohammed Attafi, Magbool Essa Oraiby, Mohammad Ahmad Attafi, Ali Mousa Sulaiman Muyidi, Hassan Abdu Ahmed Dohali. Study the Relation Between Acetylcholinesterase and Obesity in University Students. International Journal of Nutrition and Food Sciences. Vol. 8, No. 3, 2019, pp. 46-51. doi: 10.11648/j.ijnfs.20190803.11

Received: July 31, 2019; Accepted: August 20, 2019; Published: September 3, 2019

\begin{abstract}
Obesity is described as an asymmetrical body weight for height with an extreme growth of adipose tissue that is usually with the highest risk of disordered lipid profile. Obesity is caused by a sequence of excessive food intake, absence of physical motion, and hereditary predisposition. A few instances are caused primarily by genes, endocrine dysfunctions, medicines, or mental confusion. BMI gives a simple numeric test of obesity. BMIs below $20.0 \mathrm{~kg} / \mathrm{m}^{2}$ and above $25.0 \mathrm{~kg} / \mathrm{m}^{2} \mathrm{has}$ been connected with significant various health problems and most of mortality incidence causes. The recent investigations indicate a relationship between obesity, acetylcholinesterase (AChE) activities, and short-term weight loss in obese. A total of 39 female students, 18-35 years old, involved in this investigation to evaluate the relationship between body mass index (BMI), blood lipid, and AChE in female's university students. The present study reveals a strong correlation between BMI and lipid profile. As well, there is a weak negative relation between AChE and BMI in current work. But, All results of AChE in the present results within the reference range. Further long-term studies with a higher number and different types of sexes will be needed to validate and complete evaluate the specific relation between obesity and AChE.
\end{abstract}

Keywords: Obesity, Lipid Profile, BMI, Acetylcholinesterase

\section{Introduction}

Obesity is the most current nutritional disturbance in society and the vital risk factor for various related disorders [1-2]. Obesity is defined as excess body fat has grown in the body and builds a disadvantageous effect on health. Obesity is estimated for BMI and the waist-hip ratio [3-4]. A recent investigation reported that obesity could induce a definite and distinct reduction in brain size. Obesity also has been linked with significant brain atrophy, cognitive impairment, an elevation in AChE, and cholinergic response [4-6]. Accumulating proof from experimental and clinical investigations propose that imbalanced cholinergic response can drive to adverse consequences [2-4] Moreover, studies recommend that raised central sympathetic activity plays a crucial role in the etiology and complications of obesityrelated metabolic disturbances [4-5]. Therefore, getting the complex pathways underlying the metabolic disorders and treatment options to reverse the adverse metabolic phenotype has become of utmost importance [3-6]. Butyrylcholinesterase (BChE) is suspected of being involved in lipoprotein metabolism and has also been associated with the pathogenesis of the inflammatory disease, one of the potential complications related to obesity [5-7]. The recent finding indicated that short-term weight loss in obese intact female Beagle dogs resulted in opposite effects in two 
cholinesterase isoenzyme activities, specifically lower $\mathrm{BChE}$ and induce the elevation in AChE activities [3, 8-10]. In this study, we will examine to find the correlation between obesity, acetylcholinesterase, lipid profile, and BMI in females university students [10-13].

\section{Subjects and Methods}

\subsection{Subjects}

A total of 39 female students (18-35 years old) were involved in this investigation. The evaluation takes place in the period 2018-2019. Ethical approval of all procedures performed in this study was approved by the institutional research committees in both participating hospitals, university, and accordance with the ethical standards of the 1964 Helsinki Declaration 2008. Informed consent was collected from all individual participants incorporated in the study. Written consent sign by each participant after a full description of the method of the investigation. All students have the freedom to leave the study without any reason and warning of a trial.

\subsection{Methods}

\subsubsection{Anthropometrics}

Weight and height were collected with a digital medical. BMI was determined using weight (in kilograms) divided by the height squared (in square meter). Ideal body weight (IBW) was studied as the weight for BMI $=25 \mathrm{~kg} / \mathrm{m}^{2}[14]$.

\subsubsection{Laboratory Investigations}

\section{i. Biochemical tests}

All-blood tests were drawn following a 12-h fast and included lipids profile. Total cholesterol, triglyceride, highdensity lipoprotein (HDL cholesterol), low-density lipoprotein (LDL cholesterol) was measured in Abu-Arish general hospital Jazan in Saudi Arabia using Hitachi automatic analyzer c501 biochemistry.

ii. Serum AChE analysis

Serum specimens were frozen at $-80^{\circ} \mathrm{C}$ until $\mathrm{AChE}$ investigation. The activity of $\mathrm{AChE}$ was determined by the kinetic colorimetric method according to Deutsche (1992) on the ARCHITECT cSystems [15]. The method uses butyrythiocholine as the specific substrate for cholinesterase (CHE) that catalyzes the hydrolysis of butyrythiocholine substrate forming butyrate and thiocholine. Thiocholine reduces hexacyanoferrate (III) to hexacyanoferrate (II). The diminished in absorbance is directly proportional to the CHE activity in the sample. The experimental assay was run on the ARCHITECT cSystems in Poison Control and Medical Forensic Chemistry Center, Ministry of Health, Jazan, Kingdom of Saudi Arabia [15].

\subsubsection{Statistic}

Data were supplied to the computer and examined using IBM SPSS software package version 20.0. (Armonk, NY: IBM Corp). The Kolmogorov-Smirnov, Shapiro and D'agstino tests were applied to confirm the normality of distribution of variables, comparisons between groups for categorical variables were evaluated using Chi-square test (Fisher or Monte Carlo). ANOVA was applied to compare between more than two groups for normally distributed quantitative variables, and observed by Post Hoc test (Tukey) for pairwise comparison. Pearson coefficient was applied to correlate between quantitative variables. The significance of the achieved results was concluded at the $5 \%$ level.

\section{Result}

Table 1 outlined the data and results of the correlation between BMI and various parameters. According to Evans (1996) who submits for the absolute value of r: 0.00-0.19: "very weak", 0.20-0.39: "weak", 0.40-0.59: "moderate", 0.60-0.79: "strong", 0.80-1.0: "very strong". There is a positive correlation between triglyceride, LDL, and BMI as well a negative correlation between HDL and BMI. As well, there is a very weak correlation between Cholinesterase, (AChE, and BMI (table 1, figures 1-3).

Table 1. Correlation between BMI and parameters $(n=39)$.

\begin{tabular}{lll}
\hline & BMI $\left(\mathbf{k g} / \mathbf{m}^{2}\right)$ & \\
\cline { 2 - 3 } & $\mathbf{R}$ & $\mathbf{p}$ \\
\hline Cholesterol $(\mathrm{mmol} / \mathrm{L})$ & 0.003 & 0.986 \\
Triglyceride $(\mathrm{mmol} / \mathrm{L})$ & $0.331^{*}$ & $0.040^{*}$ \\
HDL $(\mathrm{mmol} / \mathrm{L})$ & $-0.577^{*}$ & $<0.001^{*}$ \\
LDL $(\mathrm{mmol} / \mathrm{L})$ & $0.343^{*}$ & $0.033^{*}$ \\
Cholinesterase $(\mathrm{U} / \mathrm{L})$ & -0.120 & 0.468 \\
\hline
\end{tabular}

R: Pearson coefficient.

*: Statistically significant at $\mathrm{p} \leq 0.05$.

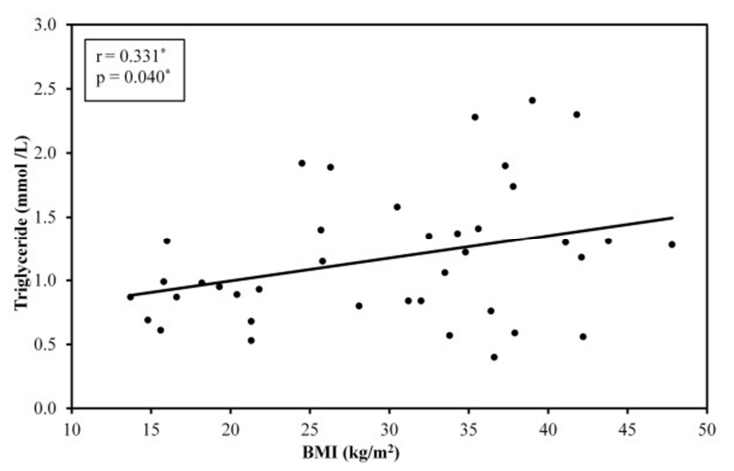

Figure 1. Correlation between BMI $\left(\mathrm{kg} / \mathrm{m}^{2}\right)$ and Triglyceride ( $\left.\mathrm{mmol} / \mathrm{L}\right)$ $(n=39)$.

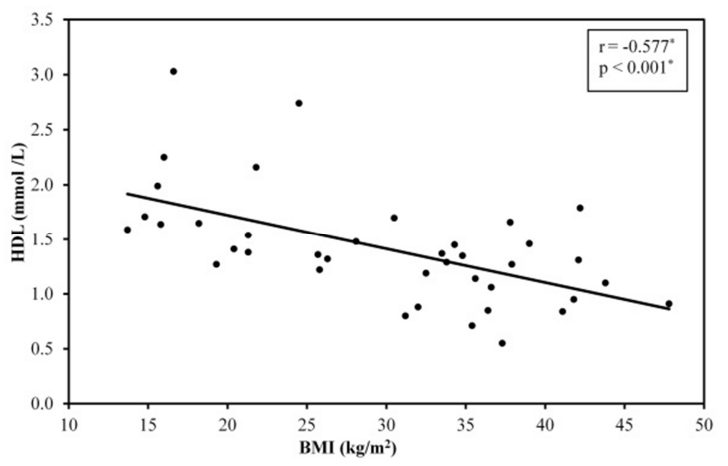

Figure 2. Correlation between BMI $\left(\mathrm{kg} / \mathrm{m}^{2}\right)$ and $H D L(\mathrm{mmol} / \mathrm{L})(\mathrm{n}=39)$. 


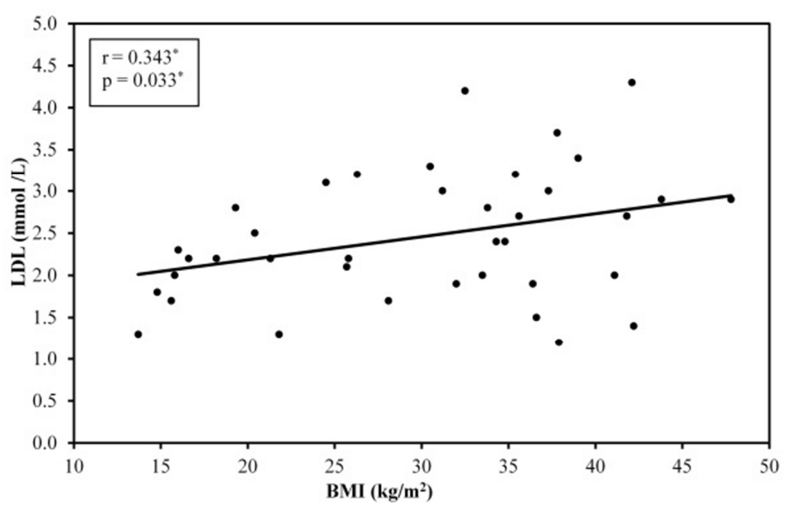

Figure 3. Correlation between BMI $\left(\mathrm{kg} / \mathrm{m}^{2}\right)$ and $L D L(\mathrm{mmol} / \mathrm{L})(n=39)$.

Table 2 represented the relation between BMI type and lipid profile parameters as well cholinesterase, the data described as Mean \pm SD. Serum cholesterol dates were represented as Mean $\pm \mathrm{SD}(4.1 \pm 0.8,4.3 \pm 1.0,3.9 \pm 0.7,4.1 \pm 1.0)$ in underweight, normal weight, overweight, obesity groups respectively as compared to each other while normal range (4-6.8mmol/L), figure 4 . Triglyceride levels in serum were represented as Mean \pm SD. The results showed $(0.9 \pm 0.2$, $1.0 \pm 0.5,1.3 \pm 0.5,1.3 \pm 0.6$ ) in underweight, normal weight, overweight, obesity groups respectively as compared to each other while normal range (1-1.2 mmol/L), figure 4. Serum $\mathrm{HDL}$ were represented as Mean $\pm \mathrm{SD}$ in different studied groups $(2.0 \pm 0.5,1.8 \pm 0.6,1.4 \pm 0.1,1.2 \pm 0.3)$ in underweight, normal weight, overweight, obesity groups respectively as compared to each other while normal range (1-2.2mmol/L). LDL levels in serum were represented as Mean \pm SD in different studied groups $(1.9 \pm 0.4,2.4 \pm 0.6,2.3 \pm 0.6,2.7 \pm 0.8)$ in underweight, normal weight, overweight, obesity groups respectively as compared to each other while normal range $(1.8-4.5 \mathrm{mmol} / \mathrm{L})$, figure 4 . Cholinesterase levels in serum were represented as Mean $\pm \mathrm{SD}$ in different studied groups $(8060 \pm 1674, \quad 7887 \pm 1496, \quad 8992 \pm 1453, \quad 7534 \pm 1238) \quad$ in underweight, normal weight, overweight, obesity groups respectively as compared to each other while normal range (4389-10928U/L), figure5. Table 3 represents the percent of normal case of lipid profile parameters as well cholinesterase in different studied group, figures 6, 7 .

Table 2. Relation between BMI type and parameters $(n=39)$.

\begin{tabular}{|c|c|c|c|c|c|c|}
\hline & \multicolumn{4}{|l|}{ BMI type $\left(\mathrm{kg} / \mathrm{m}^{2}\right)$} & \multirow{2}{*}{$\mathbf{F}$} & \multirow{2}{*}{$\mathbf{p}$} \\
\hline & Underweight $(n=7)$ & Normal weight $(n=6)$ & Overweight $(n=4)$ & Obesity $(n=22)$ & & \\
\hline \multicolumn{7}{|l|}{ Cholesterol $(\mathrm{mmol} / \mathrm{L})$} \\
\hline Median (Min.-Max.) & $3.9(3.1-4.5)$ & $4(3.6-6.2)$ & $3.7(3.4-4.9)$ & $4.1(2.6-5.8)$ & \multirow{3}{*}{0.158} & \multirow{3}{*}{0.924} \\
\hline Mean \pm SD & $4.1^{\mathrm{a}} \pm 0.8$ & $4.3^{\mathrm{a}} \pm 1.0$ & $3.9^{\mathrm{a}} \pm 0.7$ & $4.1^{\mathrm{a}} \pm 1.0$ & & \\
\hline Triglyceride $(\mathrm{mmol} / \mathrm{L})$ & & & & & & \\
\hline Median (Min.-Max.) & $0.9(0.6-1.3)$ & $0.9(0.5-1.9)$ & $1.3(0.8-1.9)$ & $1.3(0.4-2.4)$ & \multirow{2}{*}{1.367} & \multirow{2}{*}{0.269} \\
\hline $\begin{array}{l}\text { Mean } \pm \text { SD. } \\
\text { HDL }(\mathrm{mmol} / \mathrm{L})\end{array}$ & $0.9^{\mathrm{a}} \pm 0.2$ & $1.0^{\mathrm{a}} \pm 0.5$ & $1.3^{\mathrm{a}} \pm 0.5$ & $1.3^{\mathrm{a}} \pm 0.6$ & & \\
\hline Median (Min.-Max.) & $1.7(1.6-3)$ & $1.5(1.3-2.7)$ & $1.3(1.2-1.5)$ & $1.2(0.6-1.8)$ & \multirow{2}{*}{$8.834^{*}$} & \multirow{2}{*}{$<0.001^{*}$} \\
\hline $\begin{array}{l}\text { Mean } \pm \text { SD. } \\
\operatorname{LDL}(\mathrm{mmol} / \mathrm{L})\end{array}$ & $2.0^{\mathrm{a}} \pm 0.5$ & $1.8^{\mathrm{a}} \pm 0.6$ & $1.4^{\mathrm{ab}} \pm 0.1$ & $1.2^{\mathrm{b}} \pm 0.3$ & & \\
\hline Median (Min.-Max.) & $2(1.3-2.3)$ & $2.4(1.3-3.1)$ & $2.2(1.7-3.2)$ & $2.8(1.2-4.3)$ & \multirow{3}{*}{1.942} & \multirow{3}{*}{0.141} \\
\hline Mean \pm SD & $1.9^{\mathrm{a}} \pm 0.4$ & $2.4^{\mathrm{a}} \pm 0.6$ & $2.3^{\mathrm{a}} \pm 0.6$ & $2.7^{\mathrm{a}} \pm 0.8$ & & \\
\hline Cholinesterase (U/L) & & & & & & \\
\hline Median (Min.-Max.) & $8288(4895-9716)$ & 8009 (5584-9868) & 9075 (7289-10528) & $7510(5658-10290)$ & \multirow{2}{*}{1.354} & \multirow{2}{*}{0.273} \\
\hline Mean \pm SD. & $8060^{\mathrm{a}} \pm 1674$ & $7887^{\mathrm{a}} \pm 1496$ & $8992^{\mathrm{a}} \pm 1453$ & $7534^{\mathrm{a}} \pm 1238$ & & \\
\hline
\end{tabular}

Underweight $=<18.5$ Normal weight $=18.5-24.9$ Overweight $=25-29.9$ Obesity=BMI of 30 or greater.

F: F for ANOVA test, Pairwise comparison bet. each 2 groups was done using Post Hoc test, (Tukey).

$\mathrm{p}$ : $\mathrm{p}$ value for comparing between the different categories.

Means with Common letters are not significant (i.e. Means with Different letters are significant).

*: Statistically significant at $\mathrm{p} \leq 0.05$.

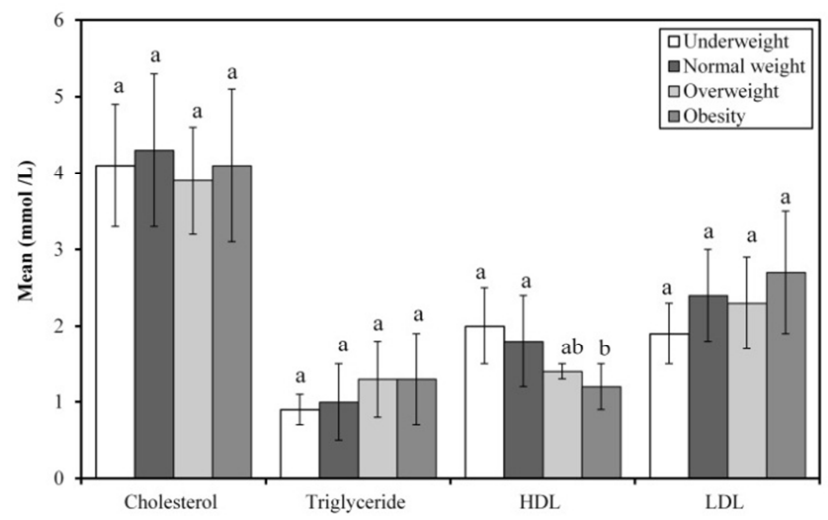

Figure 4. Relation between BMI type and lipid parameters in different studied groups $(n=39)$.

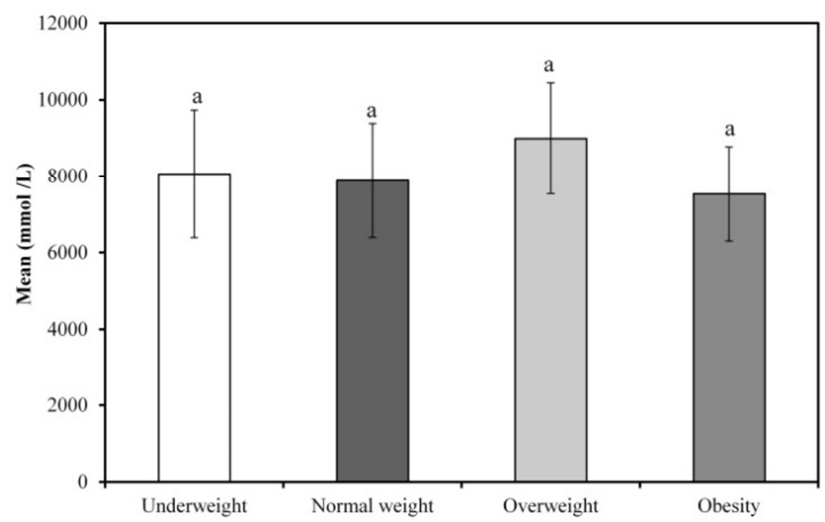

Figure 5. Relation between BMI type and Cholinesterase (U/L) in different studied group $(n=39)$. 
Table 3. The percent of normal case of different studied parameters in different studied groups $(n=39)$.

\begin{tabular}{|c|c|c|c|c|c|c|}
\hline & \multicolumn{4}{|l|}{ BMI Type } & \multirow{2}{*}{$\chi^{2}$} & \multirow[b]{2}{*}{$\mathbf{p}$} \\
\hline & Underweight $(n=7)$ & Normal weight (n=6) & Overweight $(n=4)$ & Obesity $(n=22)$ & & \\
\hline Cholesterol (mmol/L) (4-6.8) & $3(42.9 \%)$ & $3(50 \%)$ & $1(25 \%)$ & $12(54.5 \%)$ & 1.374 & 0.802 \\
\hline Triglyceride $(\mathrm{mmol} / \mathrm{L})(0.4-2.1)$ & $7(100 \%)$ & $6(100 \%)$ & $4(100 \%)$ & $19(86.4 \%)$ & 1.431 & 0.827 \\
\hline $\mathrm{HDL}(\mathrm{mmol} / \mathrm{L})(1-2.2)$ & $5(71.4 \%)$ & $5(83.3 \%)$ & $4(100 \%)$ & $14(63.6 \%)$ & 2.182 & 0.547 \\
\hline $\mathrm{LDL}(\mathrm{mmol} / \mathrm{L})(1.8-4.5)$ & $5(71.4 \%)$ & $5(83.3 \%)$ & $3(75 \%)$ & $19(86.4 \%)$ & 1.654 & 0.790 \\
\hline Cholinesterase (U/L) (4389-10928) & $7(100 \%)$ & $6(100 \%)$ & $4(100 \%)$ & $22(100 \%)$ & - & - \\
\hline
\end{tabular}

Underweight $=<18.5$ Normal weight $=18.5-24.9$ Overweight $=25-29.9$ Obesity $=$ BMI of 30 or greater.

$\chi^{2}$ : Chi square test.

$\mathrm{p}$ : $\mathrm{p}$ value for comparing between the different categories.

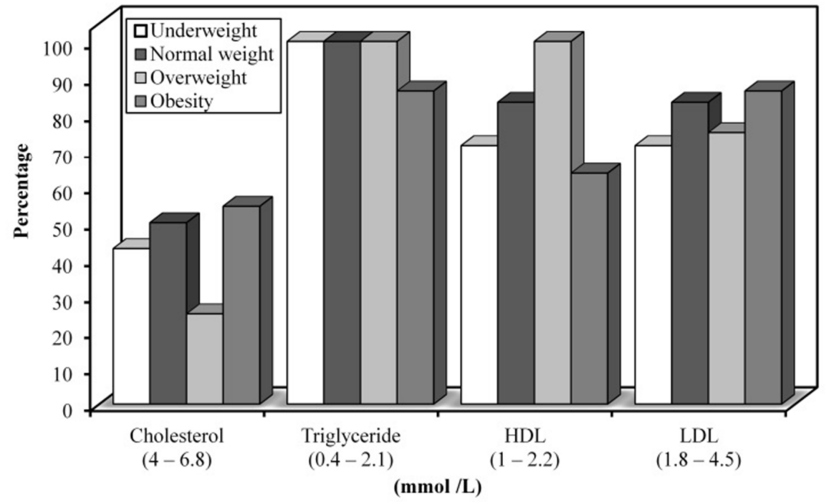

Figure 6. The percent of normal sample of lipid parameters indifferent studied groups $(n=39)$.

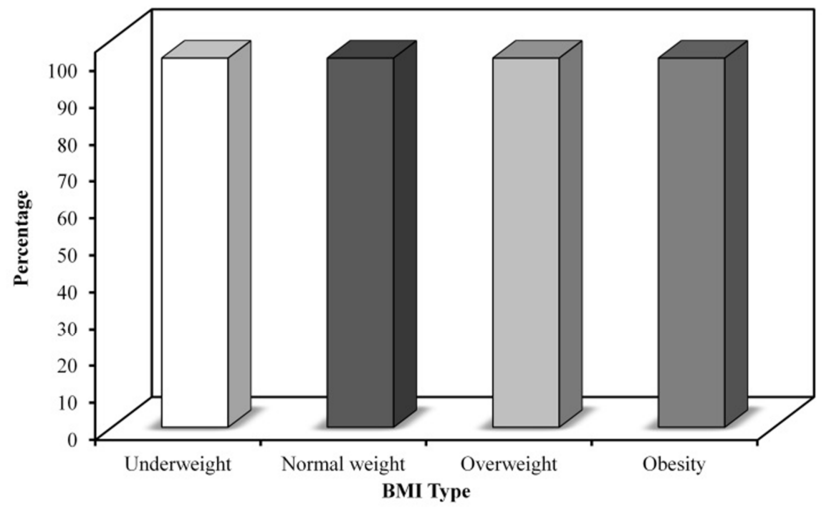

Figure 7. The percent of Cholinesterase (U/L) normal case of different studied groups $(n=39)$.

\section{Discussion}

Obesity is a chronic condition identified by excessive accumulation of body fat that is detrimental to health, as it is connected with critical metabolic complications, such as chronic inflammation, heart disorder atherosclerosis, lipid metabolic disorder and other. [16-18]. Obesity is represented as a BMI of $30 \mathrm{~kg} / \mathrm{m}^{2}$ or higher and is recognized to be one of the fastest expanding health obstacles in the modern world $[17,18]$. In this present research, there is a positive correlation between triglyceride, LDL, and BMI as well a negative association between HDL and BMI, these results agree with the previous report which confirmed that a positive relationship between lipid profile and BMI as well as a negative correlation between HDL and BMI $[17,18]$. As well, there is a very weak negative correlation between $\mathrm{AChE}$ and BMI and the data of AChE within the normal range in all studied groups. This result disagrees with previous work that showed that serum AChE activity raises with BMI in a dose-dependent and reach the plateau phase at BMIs above $30-35 \mathrm{~kg} / \mathrm{m}^{2}$ [19].

The autonomic nervous system is the division of the nervous system that regulates internal body functions as well subdivided into the sympathetic nervous system that responsible for the fight-or-flight response and the parasympathetic nervous system that functions for rest and digest as an example. Obesity is marked by the sympathetic nervous system (SNS) control in the basal state and decreased SNS responsiveness after various sympathetic stimuli $[20,21]$. In another study group of morbidly obese, the reduction in body weight was connected with a rise in the indices of parasympathetic nervous system activity, which are inversely related with sympathetic neural action and maybe imbalanced in the parasympathetic cholinergic response and inflammation [22]. Accumulating data from laboratory and clinical investigations implies that imbalanced cholinergic response can lead to adverse outcomes [23, 24]. Additionally, recent studies suggest that increased central sympathetic activity plays a pivotal role in the etiology [25] and complications [26] of obesity-related metabolic disorders. Therefore, understanding and evaluating pathways of the metabolic homeostasis in case of obesity and cholinergic response can help treatment options to reverse the adverse metabolic complication issues and has become an urgent need [27].

The parasympathetic neurotransmitter, acetylcholine (ACh), has proved an ability to prevent inflammation by inhibition of cytokine synthesis and discharge [28, 29] still, it is notably labile and challenging to serve for clinical measurements [30]. ACh is hydrolyzed by two homologous enzymes acetylcholinesterase (AChE) and butyrylcholinestersase (BChE) in the serum [16]. AChE and $\mathrm{BChE}$ have various physiological roles depending on their localization and time of expression [31]. The enzymatic activities AChE and BChE have been recommended as surrogate markers of low-grade systemic inflammation in obesity and other metabolic dysfunctions while tissuespecific, low-grade inflammation is difficult to detect and confirm [30,31]. 
Cholinesterase also, known as acetylcholine acetylhydrolase EC 3.1.1.7, is located in erythrocytes, in the lungs, spleen, and the grey matter of the brain. Pseudocholinesterase (CHE), also pointed to as acylcholine acylhydrolase EC 3.1.1.8, is detected in serum, liver, pancreas, heart, and the white matter of the brain. CHE is beneficial to diagnose liver disorders such as hepatitis, cirrhosis, carcinoma with metastasis, sensitivity to succinylcholine administration, and pesticide poisoning [15, 19]. Levels of CHE decrease in all of the diseases above. On the other hand, AChE elevation associated with obesity, metabolic syndrome, and inflammation. However, the association between morbid obesity and AChE and the alterations in cholinergic tone following bariatric laparoscopic sleeve gastrectomy (LSG) surgery-induced weight reduction never broad investigated. Recently Shenhar-Tsarfaty et al., 2018 reported that Obesity-related AChE resistance phenotype may be reversed following LSG and correlates with metabolic outcomes. They reported that further long-term studies would be needed to validate and evaluate the beneficial effect of $\mathrm{AChE}$ reduction postbariatric surgery $[15,19]$.

The pharmacological and toxic actions of AChE inhibitors consist in inactivation of the enzyme activity following an increase of synaptic $\mathrm{ACh}$ and consequently improved stimulation of postsynaptic cholinergic receptors in the central and peripheral nervous systems. According to the method of action, AChE inhibitors can be arranged into two groups irreversible and reversible. Reversible, competitive or non-competitive, inhibitors are protagonists in the pharmacotherapy of Alzheimer's disorder signs. Their therapeutic outcome is based on managing the ACh level by slowing down its hydrolysis rate $[17,31]$. Therefore, these frequently well-tolerated drugs enhance cholinergic doing so neurotransmission in forebrain areas and compensate for the loss of working brain cells. On the other hand, a previous investigation report that obesity is linked to brain shrinkage and memory deficits and may participate in the development of neurodegenerative conditions such as Alzheimer's Disease $[2,17,18,31]$.

The present study finds a weak negative correlation between AChE and obesity disorder. However, the present study has numerous limitations. First, we did not make follow up studies for weight loss and weight gain as previous investigations. So, we require that the follow-up period is a one-year weight loss process and one-year weight regains process. Second, the data were possible only for small sample numbers (39 female students). Thus, further studies with higher sample numbers as well both different-sexes male and female will be needed to evaluate if there is a relationship between AChE activity and obesity or not. Thus, more investigation will be helpful in the treatment of obesity neuroinflammatory diseases.

\section{Conclusion}

In conclusion, there is a positive correlation between triglyceride, LDL, and BMI as well a negative association between HDL and BMI in the present work. The current study also reveals that AChE has a weak negative relationship with obesity in female university students that will need further investigation and inquires to offer a new treatment of brain, obesity, and metabolic disorder.

\section{Conflict of Interest}

The authors declare that they have no conflict of interest.

\section{Acknowledgements}

The authors thank Taymour-Lank M. Farawilla and doctors in helping this work. The research community wants to thank all students who trusted us and were honest with us, which made the research more accurate and useful.

\section{References}

[1] Pêgo-Fernandes PM, Bibas BJ, Deboni M (2011). Obesity: the greatest epidemic of the 21st century? Sao Paulo Med J; 129 (5): 283-4.

[2] Hamouda FH (2016). The Association between Lifestyle, Anthropometric Measurements, and Obesity in University Students. Journal of Pharmacy and Pharmacology 4, 119-127.

[3] Abdul Rahman Al-Ajlan (2011). Lipid Profile in Relation to Anthropometric Measurements among College Male Students in Riyadh, Saudi Arabia: A Cross-Sectional Study. journal of Biomedical science. (Int J Biomed Sci; 7 (2): 112-119).

[4] Tvarijonaviciute Al, Ceron JJ, Tecles F (2013). Acetylcholinesterase and butyrylcholinesterase activities in obese Beagle dogs before and after weight loss. Vet Clin Pathol; 42 (2): 207-11.

[5] Ward MA, Carlsson CM, Trivedi MA, Sager MA, Johnson SC (2005). The effect of body mass index on global brain volume in middle-aged adults: a cross sectional study. BMC Neurol; 5: 23 .

[6] Debette S, Seshadri S, Beiser A, et al (2011). Midlife vascular risk factor exposure accelerates structural brain aging and cognitive decline. Neurology; 77 (5): 461-8.

[7] Enzinger C, Fazekas F, Matthews PM, et al (2005). Risk factors for progression of brain atrophy in aging: six-year follow-up of normal subjects. Neurology; 64 (10): 1704-11.

[8] Pannacciulli N, Del Parigi A, Chen K, Le DS, Reiman EM, Tataranni PA (2006). Brain abnormalities in human obesity: a voxel-based morphometric study. Neuroimage; 31 (4): 1419-25.

[9] Bolzenius JD, Laidlaw DH, Cabeen RP, et al (2013). Impact of body mass index on neuronal fiber bundle lengths among healthy older adults. Brain Imaging Behav (3): 300-6.

[10] Stanek KM, Strain G, Devlin M, et al (2013). Body mass index and neurocognitive functioning across the adult lifespan. Neuropsychology; 27 (2): 141-51.

[11] Ford ES, Mokdad AH, Giles WH (2003). Trends in waist circumference among U.S. adults. Obes Res; 11 (10): 1223-31. 
[12] Whitmer RA, Gustafson DR, Barrett-Connor E, Haan MN, Gunderson EP, Yaffe K (2008). Central obesity and increased risk of dementia more than three decades later. Neurology; 71 (14): 1057-64.

[13] Jagust W, Harvey D, Mungas D, Haan M (2005). Central obesity and the aging brain. Arch Neurol; 62 (10): 1545-8.

[14] Toolabi K, Arefanian S, Golzarand M, Arefanian H (2011). Effects of laparoscopic Roux-en-Y gastric bypass (LRYGB) on weight loss and biomarker parameters in morbidly obese patients: a 12-month follow-up. Obes Surg; 21: 1834-42.

[15] Deutsche GKC (1992). Proposal of standard methods for the determination of enzyme catalytic concentrations in serum and plasma at 37C. II Cholinesterase (acylcholine acylhydrolase, EC 3.1.1.8). Eur J Clin Chem Clin Biochem; 30: 163.

[16] Cruz KJ, de Oliveira AR, Morais JB, et al (2017). Role of microRNAs on adipogenesis, chronic low-grade inflammation, and insulin resistance in obesity. Nutrition; 35: $28-35$.

[17] Hamouda AF, Abou El Noeman SA (2016). Effects of 6Month Weight Loss New Program on Anthropometric Measurements and Biological Profile. Journal of Pharmacy and Pharmacology (4) 23-38.

[18] Hamouda AF, Abou El Noeman SA, Khardalic IA, et al (2018). Study the Association Between Diet Program on Human Semen, Biological Profile, and Anthropometric Measurements in Obese Men. International Journal of Nutrition and Food Sciences; 7 (1): 24-29.

[19] Shani ST, Shiri SD, Galia B, et al. (2019) Obesity-related acetylcholinesterase elevation is reversed following laparoscopic sleeve gastrectomy (2018). Int J Obes (Lond); 43 (2): 297-305.

[20] Tentolouris N, Liatis S, Katsilambros N (2006). Sympathetic system activity in obesity and metabolic syndrome. Ann N Y Acad Sci; 1083: 129-52.
[21] Poirier P, Hernandez TL, Weil KM, Shepard TJ, Eckel RH (2003). Impact of diet-induced weight loss on the cardiac autonomic nervous system in severe obesity. Obes Res; 11: 1040-7.

[22] Karason K, Molgaard H, Wikstrand J, Sjostrom L (1999). Heart rate variability in obesity and the effect of weight loss. Am J Cardiol; 83: 1242-7.

[23] Ofek K, Soreq H (2013). Cholinergic involvement and manipulation approaches in multiple system disorders. ChemBiol Interact; 203: 113-9.

[24] Shenhar-Tsarfaty S, Berliner S, Bornstein NM, Soreq H (2014). Cholinesterases as biomarkers for parasympathetic dysfunction and inflammation-related disease. J Mol Neurosci; 53: 298-305.

[25] Myers MG, Olson DP (2012). Central nervous system control of metabolism. Nature; 491: 357-63.

[26] Thorp AA, Schlaich MP (2015). Relevance of sympathetic nervous system activation in obesity and metabolic syndrome. J Diabetes Res: 341583.

[27] Metz CN, Tracey KJ (2005). It takes nerve to dampen inflammation. Nat Immunol; 6: 756-57.

[28] Borovikova LV, et al (2000). Vagus nerve stimulation attenuates the systemic inflammatory response to endotoxin. Nature; 405: 458-62.

[29] Soreq H, Seidman S (2001). Acetylcholinesterase--new roles for an old actor. Nat Rev Neurosci; 2: 294-302.

[30] Das UN (2007). Acetylcholinesterase and butyrylcholinesterase as possible markers of low-grade systemic inflammation. Med Sci Monit; 13: Ra214-21.

[31] Mirjana BO, Danijela ZK, Tamara DL, et al (2013). Acetylcholinesterase Inhibitors: Pharmacology and Toxicology Current Neuropharmacology, 11, 315-335 315. 